\title{
Some useful techniques for pointwise and local error estimates of the quantities of interest in the finite element approximation
}

\author{
T. Cao* D. W. Kelly ${ }^{\dagger} \quad$ M. Ainsworth ${ }^{\ddagger}$ \\ (Received 7 August 2000)
}

\begin{abstract}
In this paper we review some existing techniques to obtain pointwise and local a posteriori error estimates for the quantities of interest in finite element approximations by using duality arguments. We

* School of Mathematics, University of New South Wales, Sydney 2052, Australia.

$\dagger$ School of Mechanical and Manufacturing Engineering, University of New South Wales, Sydney 2052, Australia.

$\ddagger$ Department of Mathematics, University of Strathclyde, Glasgow, Scothand.

${ }^{0}$ See http://anziamj.austms.org.au/V42/CTAC99/Cao for this article and ancillary services, (c) Austral. Mathematical Soc. 2000. Published 27 Nov 2000.
\end{abstract}


also present a new approach to obtain computable error bounds for the recovered pointwise quantities. The new method is extended to include the practically important case of non-homogeneous Dirichlet data. Existing methods require purely Neumann data, or the Dirichlet data to be homogeneous. The new techniques are developed here to provide computable error bounds on the genuine pointwise quantities and allow the use of non-homogeneous Dirichlet data. The strength and weakness of each technique will be analysed and compared. The numerical experiments to justify our analysis will be presented.

\section{Contents}

1 Introduction

C319

2 Bounding techniques using auxiliary problems

C321

3 Error bounds for pointwise derivatives using a modified Green function

C322

4 Estimate errors in energy for non-homogeneous Dirichlet BVPs

C325

5 Error bounds for pointwise "ball recovery" derivative values

C328 


\section{Introduction}

For most engineering analysis and design, pointwise and local quantities related to stress and displacements are of primary concern. Thus, it is important to have an effective control of these quantities, which are usually produced by the finite element method. In this paper, we will study a number of approaches using duality arguments and newly defined auxiliary problems to produce a posteriori error estimates for these finite element calculations. We can categorise these approaches into two different groups.

In the first group (see [7] and [4]), an a posteriori error estimate for pointwise finite element solutions were obtained by using a modified Green function for the auxiliary problem. This approach is quite simple and inexpensive in terms of computational costs, and applicable to a very wide range of problems. It is very useful for designing a mesh adaptive algorithm. However, due to the singularity of the Green function (particularly for the derivative) the pointwise error estimate obtained by this approach dramatically overestimates the true error. A more expensive approach to seek a high quality error bound has been proposed by the second group (see [8] and [3]). In this approach, the accurate recovery of pointwise quantities from finite 
element solutions using boundary integrals, and a posteriori error bounds for the recovered quantities, have been obtained. The recovered pointwise quantities are always more accurate than the finite element quantities, and the upper error bounds for these recovered quantities are computable, tight and reliable. The limitations of this approach are that the computational cost is high, and it is only applicable for purely traction (purely Neumann) boundary conditions. For Dirichlet boundary condition the approach has to be modified. In this paper, we present a new approach employing the "ball recovery" techniques introduced in [6] and the equilibration technique proposed in [1]. Here, the equilibration techniques are extended to include the case of non-homogeneous Dirichlet boundary data (see [2]). This new approach can apply to any mesh and any finite element solution for nonhomogeneous Dirichlet boundary value problems. It proves to be very useful for recovering pointwise values from finite element solutions, and at obtaining a posteriori error bounds for these recovered quantities.

The outline of this paper is as follows. In the next section, we introduce the general techniques for bounding the "quantities of interest" via an auxiliary problem. In Section 3, we review the a posteriori error estimates for the direct local and pointwise finite element calculations. In Section 4, we present the equilibration techniques modified for the non-homogeneous Dirichlet boundary problems. The modified "ball recovery" techniques for the pointwise values at the interior points and the points close to or on the boundary and their error bounds are introduced in Section 5. The numerical experiments are presented in Section 6. 


\section{Bounding techniques using auxiliary prob- lems}

For the presentation of the theory, we consider an abstract problem. Let $\Omega$ be an bounded domain in $\mathbb{R}^{d}$ with boundary $\Gamma$. $V$ is assumed to be a Hilbert space of functions defined on $\Omega$. The model problem consist of finding a function $u \in V$ which satisfies

$$
B(u, v)=F(v), \quad \forall v \in V,
$$

$B(\cdot, \cdot)$ is a symmetric positive definite bilinear form on $V \times V$ and thus defines an inner product on $V$. The loading $F$ is an element of the dual space $V^{\prime}$. The boundary conditions satisfied by $u$ on $\Gamma$ are implicitly included in the definition of the space $V$ and the loading $F$. Then by the Lax-Milgram theorem, Problem (1) admits a unique solution $u \in V$. Let $V_{h} \subset V$ be the finite element space of piecewise polynomial functions. The mesh $\mathcal{P}$, formed by the union of all elements $K$, is assumed to coincide exactly with the domain $\Omega$. By the classical Galerkin method, there exists a finite element solution $u_{h} \in V_{h}$ such that

$$
B\left(u_{h}, v_{h}\right)=F\left(v_{h}\right), \quad \forall v_{h} \in V_{h} .
$$

Let $J(\cdot)$ be an arbitrary linear functional defined on $V$ and let $e=u-u_{h}$ be the error between the exact solution $u$ and the finite element solution $u_{h}$. We are interested in bounding the quantity $J(e)=J(u)-J\left(u_{h}\right)$. Our bounding technique is as below. 


\section{Error bounds for pointwise derivatives using a modified Green function C322}

We consider the auxiliary problem: find $g \in V$ such that

$$
B(g, v)=J(v), \quad \forall v \in V .
$$

The corresponding finite element problem is: find $g_{h} \in V_{h}$ such that

$$
B\left(g_{h}, v_{h}\right)=J\left(v_{h}\right), \quad \forall v_{h} \in V_{h} .
$$

Using orthogonality we have

$$
\begin{aligned}
J(u)-J\left(u_{h}\right) & =B\left(u-u_{h}, g-g_{h}\right)=\sum_{K \in \mathcal{P}} B_{K}\left(u-u_{h}, g-g_{h}\right) \\
& \leq \sum_{K \in \mathcal{P}}\left\|u-u_{h}\right\|_{B_{K}}\left\|g-g_{h}\right\|_{B_{K}} \leq \sum_{K \in \mathcal{P}} \gamma_{K} \eta_{K},
\end{aligned}
$$

where $B_{K}$ is the restriction of $B$ on element $K,\|\cdot\|_{B_{K}}=B_{K}(\cdot, \cdot)^{1 / 2}$, and $\gamma_{K}, \eta_{K}$ are a posteriori local error estimates for $\left\|u-u_{h}\right\|_{B_{K}},\left\|g-g_{h}\right\|_{B_{K}}$ respectively (see [1] and [4]). These local a posteriori error estimates are crucial for the design of reliable adaptive methods to improve the efficiency of modelling and simulation.

\section{Error bounds for pointwise derivatives us- ing a modified Green function}

This approach was proposed in [4] and [7]. We consider the following problem

$$
\begin{array}{rll}
-\Delta u=f & \text { in } \Omega \subset \mathbb{R}^{2}, \\
u=0 & \text { on } \Gamma,
\end{array}
$$




\section{Error bounds for pointwise derivatives using a modified Green function C323}

where $\Omega$ is a bounded domain and $\Gamma$ is its boundary, and $f \in L^{2}(\Omega)$. The weak solution is $u \in H_{0}^{1}(\Omega)$ such that

$$
B(u, v)=\langle f, v\rangle, \quad \forall v \in H_{0}^{1}(\Omega),
$$

where $B(u, v)=\int_{\Omega} \nabla u \nabla v d \mathbf{x}$ and $\langle f, v\rangle=\int_{\Omega} f v d \mathbf{x}$ and $\mathbf{x}=(x, y) \in \Omega$.

The technique for bounding a pointwise derivative value is as below (a similar and simpler technique was used for bounding a pointwise displacement value).

Let $\mathbf{x}_{0}=\left(x_{0}, y_{0}\right) \in \Omega$ be point of interest and $J(u)=\frac{\partial u}{\partial x}$ be the quantity of interest, we consider the auxiliary problem:

Find the dual solution $g$ such that

$$
B(g, v)=\left\langle-\frac{\partial \delta_{\mathbf{x}_{0}}}{\partial x}, v\right\rangle=\frac{\partial v}{\partial x}\left(\mathbf{x}_{0}\right)=J(v),
$$

where $\delta_{\mathbf{x}_{0}}$ is the Dirac Delta Function with point loaded at $\mathbf{x}_{0}$. However $g=O\left(\left|\mathbf{x}-\mathbf{x}_{0}\right|^{-1}\right)$ (see [5]) which does not belong to $H_{0}^{1}(\Omega)$. The problem encountered can be overcome by modifying the dual solution $g$ in a number of different approaches, as proposed in [4] and [7].

In order to obtain the error estimate for the pointwise quantity $J(u)=$ $\frac{\partial u}{\partial x}\left(\mathbf{x}_{0}\right)$, it was suggested in [4] to regularise the functional $J(u)$ by defining $J_{\epsilon}(u)=\frac{1}{\left|B_{\epsilon}\left(\mathbf{x}_{0}\right)\right|} \int_{B_{\epsilon}\left(\mathbf{x}_{0}\right)} \frac{\partial u}{\partial x} d \mathbf{x}$, where $B_{\epsilon}\left(\mathbf{x}_{0}\right)$ is a ball centered at $\mathbf{x}_{0}$ with radius $\epsilon$. Therefore

$$
\left|J(u)-J_{\epsilon}(u)\right|=O(\epsilon)
$$




\section{Error bounds for pointwise derivatives using a modified Green function C324}

and the corresponding dual solution $g_{\epsilon}(\mathbf{x}) \approx \frac{x-x_{0}}{\left(\left|\mathbf{x}-\mathbf{x}_{0}\right|+\epsilon\right)^{2}}$ is well defined in $H_{0}^{1}(\Omega)$. The meshes used in [4] and in our numerical experiment are constructed such that $\mathbf{x}_{0}$ does not lie on the element boundaries and therefore $J\left(u_{h}\right)$ is well defined. Hence $J(e)$ can be approximately bounded by:

$$
\left|J(u)-J\left(u_{h}\right)\right| \approx\left|J_{\epsilon}(u)-J_{\epsilon}\left(u_{h}\right)\right| \leq C_{i} \sum_{K \in \mathcal{P}} \omega_{K}^{\epsilon} \rho_{K},
$$

where $C_{i}=C_{1} C_{2}$ and $C_{1}, C_{2}$ are interpolation constants arising from $u$ and $g$ respectively, and

$$
\omega_{K}^{\epsilon}=\frac{h_{K}^{2}}{\left(r_{K}+\epsilon\right)^{3}} \quad \text { and } \quad \rho_{K}=h_{K}\left\|f+\Delta u_{h}\right\|_{K}+\frac{1}{2} h_{K}^{1 / 2}\left\|\mathbf{n} \cdot\left[\nabla u_{h}\right]\right\|_{\partial K},
$$

where $h_{K}=\operatorname{Diam}(K), r_{K}=\max \left\{\operatorname{dist}\left(\mathrm{K}, \mathbf{x}_{0}\right), \mathrm{h}_{\mathrm{K}}\right\}, \mathbf{n}$ is the normal vector on $\partial K$, and $\left[\nabla u_{h}\right]=\left.\frac{\partial u_{h}}{\partial \mathbf{n}}\right|_{K}-\left.\frac{\partial u_{h}}{\partial \mathbf{n}}\right|_{J}$ with $J$ being an adjacent element of $K$. The error estimate (10) depends on the unknown interpolation constant $C_{i}$ and the behaviour of $g-g_{h}$. The effectivity index (Index $=$ Estimated Error/True Error) is significantly increased when the meshsize $h$ is reduced as shown by the numerical results in [4] and in our numerical experiments. Therefore this error estimate can only be recommended for guiding adaptive mesh refinement. 


\section{Reliable and efficient a posteriori estimates for error in energy for non-homogeneous Dirichlet boundary value problems}

We consider the following problem

$$
\begin{aligned}
-\Delta u & =f \quad \text { in } \Omega, \\
u & =u_{0} \quad \text { on } \Gamma,
\end{aligned}
$$

where $u_{0} \in C(\Gamma)$ and $f \in L_{2}(\Omega)$ are given data, with $\Omega$ a planar, polygonal domain, and $\Gamma$ is its boundary. Suppose we approximate this problem by using a piecewise bilinear finite element scheme on quadrilateral elements in the usual way. The non-homogeneous Dirichlet data is approximated by the linear interpolant $u_{0}^{h}$ such that $u_{0}^{h}(\mathbf{x})=u_{0}(\mathbf{x})$ whenever $\mathbf{x}$ is a node of the element edge lying in the boundary $\Gamma$. The finite element approximation is then:

Find $u_{h} \in V_{h}$ such that

$$
\begin{aligned}
B\left(u_{h}, v_{h}\right) & =\left\langle f, v_{h}\right\rangle \quad \forall v_{h} \in V_{h}^{0}, \\
u_{h} & =u_{0}^{h} \quad \text { on } \Gamma,
\end{aligned}
$$

where $V_{h}$ is the bilinear finite element space and $V_{h}^{0}=H_{0}^{1}(\Omega) \cap V_{h}$. The error $e=u-u_{h}$ will not vanish on the boundary unless $u_{0}^{h}=u_{0}$ on $\Gamma$. Therefore, 
in order to obtain an a posteriori error bound, we decompose the error into two parts as follows:

$$
e=e_{0}+\bar{e}
$$

where $e_{0} \in H_{0}^{1}(\Omega)$ satisfies

$$
B\left(e_{0}, v\right)=B(e, v) \quad \forall v \in H_{0}^{1}(\Omega),
$$

and $\bar{e} \in H^{1}(\Omega)$ satisfies

$$
\begin{aligned}
B(\bar{e}, v) & =B(e, v)-B\left(e_{0}, v\right)=0 \quad \forall v \in H_{0}^{1}(\Omega), \\
\bar{e} & =u_{0}-u_{0}^{h} \quad \text { on } \Gamma .
\end{aligned}
$$

Each component of the error is estimated separately.

Let $K$ be any element with at least one face in $\Gamma$. That is, $\partial K \cap \Gamma$ is non-empty. Define $\Psi_{K} \in H^{1}(K)$ by

$$
\Psi_{K}=\left\{\begin{array}{l}
\bar{e} \text { on } \partial K \cap \Gamma \\
0 \text { on } \partial K \backslash \Gamma,
\end{array}\right.
$$

with

$$
B_{K}\left(\Psi_{K}, v\right)=0 \quad \forall v \in H_{0}^{1}(K),
$$

where $B_{K}\left(\Psi_{K}, v\right)=\int_{K} \nabla \Psi_{K} \nabla v d \mathbf{x} . \Psi_{K}$ are defined to be zero in the inner elements. Observe that $\bar{e}=u_{0}-u_{0}^{h}$ on $\Gamma$, vanishes at the nodes of element $K$ which are on the boundary $\Gamma$, so that (19) means $\Psi_{K}$ is continuous on $\partial K$. 
Introducing $\varphi_{K} \in H_{E}^{1}(K)$ with $K$ being any element in $\mathcal{P}$ :

$$
B_{K}\left(\varphi_{K}, v\right)=(f, v)_{K}-B_{K}\left(u_{h}, v\right)+\int_{\partial K} t_{K} v d s \quad \forall v \in H_{E}^{1}(K)
$$

where $(f, v)_{K}=\int_{K} f v d \mathbf{x}$ and $t_{K}$ is the equilibrated element boundary fluxes constructed in [1] for homogeneous Dirichlet boundary data, and

$$
H_{E}^{1}(K)=\left\{v \in H^{1}(K): v=0 \text { on } \partial K \cap \Gamma\right\} .
$$

The equilibrated boundary element fluxes $t_{K}$ are constructed such that

$$
(f, 1)_{K}-B_{K}\left(u_{h}, 1\right)+\int_{\partial K} t_{K} d s=0
$$

and therefore problem (21) is solvable. Hence $\varphi_{K}$ are not unique for inner elements but $\left\|\varphi_{K}\right\|_{B_{K}}$ are well defined.

The error components $\bar{e}$ and $e_{0}$ on the element $K$ are then estimated by $\Psi_{K}$ and $\varphi_{K}$, respectively. The error estimate for $\|e\|_{E}\left(\|\cdot\|_{E}=B(\cdot, \cdot)^{1 / 2}\right.$ denotes energy norm) is then given by the following theorem.

Theorem 1 Let $\Psi_{K}$ and $\varphi_{K}$ be defined as in (19)-(20) and (21) respectively, then

$$
\|e\|_{E}^{2} \leq \sum_{K \in \mathcal{P}}\left(\left\|\Psi_{K}\right\|_{B_{K}}^{2}+\left\|\varphi_{K}\right\|_{B_{K}}^{2}\right) .
$$

where $\|\cdot\|_{B_{K}}=B_{K}(\cdot, \cdot)^{1 / 2}$. Moreover, there exists a constant $C$ independent of $h$ such that

$$
\sum_{K \in \mathcal{P}}\left(\left\|\Psi_{K}\right\|_{B_{K}}^{2}+\left\|\varphi_{K}\right\|_{B_{K}}^{2}\right) \leq C\left(\|e\|_{E}^{2}+\sum_{K \in \mathcal{P}} h_{K}^{2}\left\|f-\bar{f}_{K}\right\|_{L_{2}(K)}^{2}\right)
$$


where $\bar{f}_{K}$ is the average value of $f$ on element $K$.

Proof: See [2].

The computation of the solutions $\varphi_{K}$ of the the local residual problems is performed in the usual fashion as for homogeneous Dirichlet data, which usually used the higher degree polynomial than the one used to construct $u_{h}$. The computation cost for $\varphi_{K}$ is low because we only have to solve (21) locally. However, in addition, one is concerned with obtaining the solution $\Psi_{K}$ of the non-homogeneous Dirichlet problem. This is achieved in the usual way and can actually be computed at no additional cost beyond the cost of solving the problems for $\varphi_{K}$, since the same stiffness matrix used to calculate $\varphi_{K}$ may be reused in the calculation.

\section{Error bounds for pointwise "ball recovery" derivative values}

The "ball recovery" technique was introduced in [6] for homogeneous Dirichlet BVP. It applies to any mesh and any finite element solution. In particular, it is very useful for recovering the values at interior points for a very wide range of problems with known fundamental solutions. For points close to or on the boundary, we have to modify the technique for problems with a non- 
homogeneous Dirichlet boundary condition. The modified and the original approaches can only apply to a convex polygonal domain. The original ball recovery technique can be described briefly as follows.

Let $\mathbf{x}_{0}=\left(x_{0}, y_{0}\right) \in \Omega$ be a fixed interior point, $B \subseteq \Omega$ be a ball centred at $\mathbf{x}_{0}$ with fixed radius $R$ ( $R$ is independent of mesh size $h$ ). The fundamental solution of the Laplace equation is

$$
\Phi_{0}\left(\mathbf{x}, \mathbf{x}_{0}\right)=-\frac{1}{\pi} \log r, \quad \mathbf{x}=(x, y) \in \Omega,
$$

where $r=\sqrt{\left(x-x_{0}\right)^{2}+\left(y-y_{0}\right)^{2}}$, and the derivatives with respect to $\mathbf{x}_{0}$ are denoted by:

$$
\Phi_{\alpha}\left(\mathbf{x}, \mathbf{x}_{0}\right)=D_{\mathbf{x}_{0}}^{\alpha} \Phi_{0}\left(\mathbf{x}, \mathbf{x}_{0}\right),
$$

where $\alpha$ is any multi-index. We now define the kernels

$$
\omega_{\alpha}\left(\mathbf{x}, \mathbf{x}_{0}\right)=\Phi_{\alpha}\left(\mathbf{x}, \mathbf{x}_{0}\right)+\psi_{\alpha}\left(\mathbf{x}, \mathbf{x}_{0}\right),
$$

where $\psi_{\alpha} \in C^{\infty}(\Omega)$ are chosen such that

$$
\omega_{\alpha}=\frac{\partial \omega_{\alpha}}{\partial \mathbf{n}}=0 \quad \text { on } \partial B
$$

where $\mathbf{n}=\left(n_{1}, n_{2}\right)$ is the normal vector on $\partial B$. Using Green's second formula and (25) we have

$$
\int_{B}\left(u \Delta \omega_{\alpha}-\omega_{\alpha} \Delta u\right) d \mathbf{x}=\int_{\partial B}\left(u \frac{\partial \omega_{\alpha}}{\partial \mathbf{n}}-\omega_{\alpha} \frac{\partial u}{\partial \mathbf{n}}\right) d \mathbf{x}=0
$$


Since $-\Delta \Phi_{\alpha}\left(\mathbf{x}, \mathbf{x}_{0}\right)=D_{\mathbf{x}_{0}}^{\alpha} \delta_{\mathbf{x}_{0}}(\mathbf{x})$, we have

$$
D^{\alpha} u\left(\mathbf{x}_{0}\right)=\int_{B} f \omega_{\alpha} d \mathbf{x}+\int_{B} u \Delta \psi_{\alpha} d \mathbf{x}
$$

Replacing $u$ by the finite element solution $u_{h}$ in (27) gives

$$
\widetilde{u}_{h}^{\alpha}\left(\mathbf{x}_{0}\right)=\int_{B} f \omega_{\alpha} d \mathbf{x}+\int_{B} u_{h} \Delta \psi_{\alpha} d \mathbf{x} .
$$

The error term is

$$
\left|D^{\alpha} u\left(\mathbf{x}_{0}\right)-\widetilde{u}_{h}^{\alpha}\left(\mathbf{x}_{0}\right)\right|=\left|\int_{B}\left(u-u_{h}\right) \Delta \psi_{\alpha} d \mathbf{x}\right|
$$

Since $\psi_{\alpha} \in C^{\infty}(\Omega)$, the order of convergence for $\widetilde{u}_{h}^{\alpha}$ will depend on finite element solution $u_{h}$. For example, if we choose $u_{h}$ to be piecewise bilinear finite element solution, then the order of convergence of the first order derivatives will be $O\left(h^{2}\right)$.

In [6], it was proposed to choose $\psi_{0}$ by

$$
\psi_{0}\left(\mathbf{x}, \mathbf{x}_{0}\right)=-\frac{1}{8 \pi R^{4}}\left(r^{4}-4 R^{2} r^{2}+(3-4 \log R) R^{4}\right),
$$

then

$$
\Delta \psi_{0}\left(\mathbf{x}, \mathbf{x}_{0}\right)=-\frac{2}{\pi R^{4}}\left(r^{2}-R^{2}\right)
$$

and $\psi_{(1,0)}$ by

$$
\psi_{(1,0)}\left(\mathbf{x}, \mathbf{x}_{0}\right)=-\frac{1}{2 \pi R^{6}}\left(x-x_{0}\right)\left(r^{4}-3 R^{2} r^{2}+3 R^{4}\right)
$$


then

$$
\Delta \psi_{(1,0)}\left(\mathbf{x}, \mathbf{x}_{0}\right)=-\frac{12}{\pi R^{6}}\left(x-x_{0}\right)\left(r^{2}-R^{2}\right),
$$

and $\psi_{(0,1)}$ can be chosen analogously as $\psi_{(1,0)}$ with $\left(x-x_{0}\right)$ replaced by $\left(y-y_{0}\right)$.

When $\mathbf{x}_{0}$ is close to or on the boundary $\Gamma$, part of the ball $B$ will lie outside the domain $\Omega$. In [6] a technique was proposed to extend continuously the solutions outside the domain when $\mathbf{x}_{0}$ is close to or on the homogeneous Dirichlet boundary. We will modify that technique to extend the solutions continuously outside the domain for a non-homogeneous Dirichlet boundary.

For simplicity, we define the domain as in Figure 1, it is possible to extend our results to convex polygonal domains. We then define $U$ as a continuously extension of $u$ into $\Omega^{*}$ by

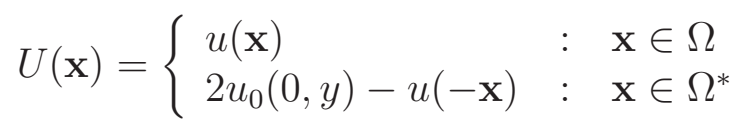

By obvious modification in the proof of (27) we have the following representation of $D^{\alpha} u\left(\mathbf{x}_{0}\right)$ for each point $\mathbf{x}_{0} \in \bar{\Omega}$ which is close to or on the boundary.

$$
\begin{aligned}
D^{\alpha} u\left(\mathbf{x}_{0}\right)= & D^{\alpha} U\left(\mathbf{x}_{0}\right)=\int_{B}-\Delta U(\mathbf{x}) \omega_{\alpha}\left(\mathbf{x}, \mathbf{x}_{0}\right) d \mathbf{x}+\int_{B} U(\mathbf{x}) \Delta \psi_{\alpha}\left(\mathbf{x}, \mathbf{x}_{0}\right) d \mathbf{x} \\
= & \int_{B_{+}} f(\mathbf{x}) \omega_{\alpha}\left(\mathbf{x}, \mathbf{x}_{0}\right) d \mathbf{x}+\int_{B_{-}}-2 \frac{\partial^{2} u_{0}}{\partial y^{2}}(0, y) \omega_{\alpha}\left(\mathbf{x}, \mathbf{x}_{0}\right) d \mathbf{x} \\
& +\int_{B_{-}} \Delta u(-x, y) \omega_{\alpha}\left(\mathbf{x}, \mathbf{x}_{0}\right) d \mathbf{x}+\int_{B_{+}} u(\mathbf{x}) \Delta \psi_{\alpha}\left(\mathbf{x}, \mathbf{x}_{0}\right) d \mathbf{x}
\end{aligned}
$$


$(0,1)$

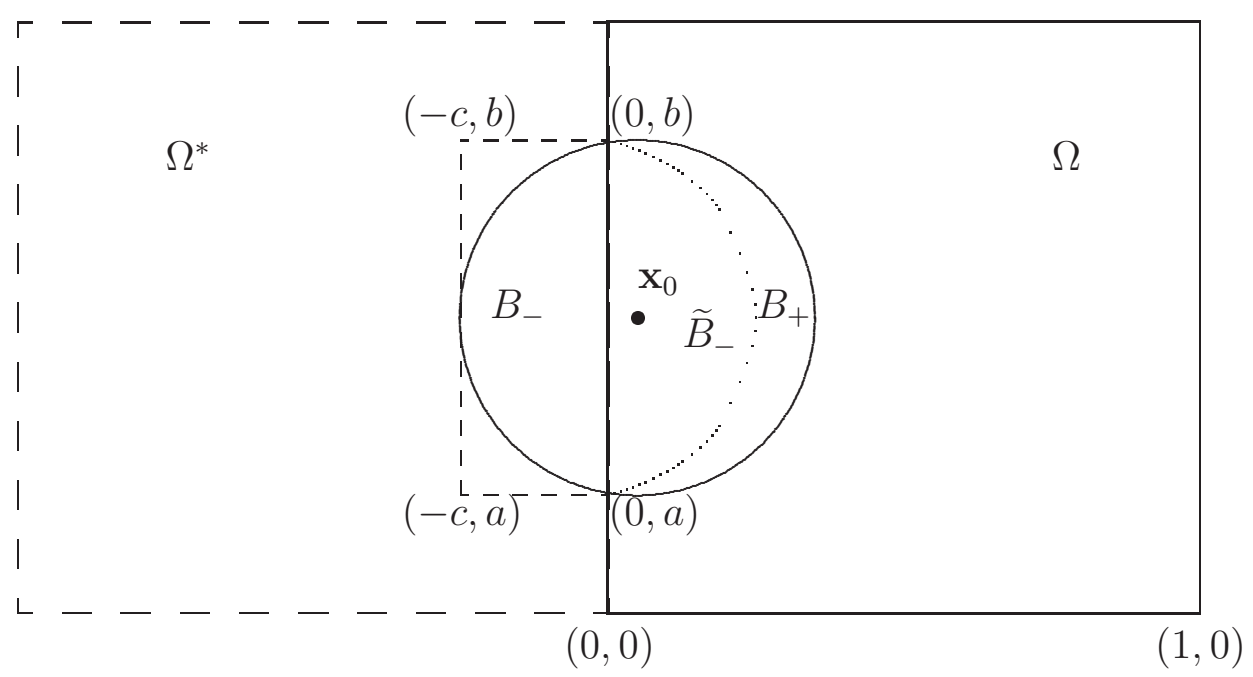

Figure 1: $\Omega=(0,1) \times(0,1), \Omega^{*}=$ reflection of $\Omega$ over the line $x=0$, $B_{+}=\Omega \cap B, B_{-}=\Omega \cap B, B=B_{+} \cup B_{-}, \widetilde{B}_{-}=$reflection of $B_{-}$over the line $x=0$. 


$$
\begin{aligned}
& +\int_{B_{-}} 2 u_{0}(0, y) \Delta \psi_{\alpha}\left(\mathbf{x}, \mathbf{x}_{0}\right) d \mathbf{x}+\int_{B_{-}}-u(-x, y) \Delta \psi_{\alpha}\left(\mathbf{x}, \mathbf{x}_{0}\right) d \mathbf{x} \\
= & I_{1}+I_{2}+I_{3}+I_{4}+I_{5}+I_{6},
\end{aligned}
$$

where $I_{1}, I_{2}, \ldots, I_{6}$ denote the previous integrands in order. It is easy to compute $I_{1}, I_{4}$. To compute $I_{3}, I_{5}, I_{6}$, we change the variables from $(x, y)$ to $(-x, y)$. To compute $I_{2}$, we use integration by parts

$$
I_{2}=\int_{B_{-}}-2 u_{0}(0, y) \frac{\partial^{2} \omega_{\alpha}\left(\mathbf{x}, \mathbf{x}_{0}\right)}{\partial y^{2}} d \mathbf{x} .
$$

The computation of $I_{2}$ will therefore depend on the smoothness of boundary condition $u_{0}$ in the neighbourhood of $\mathbf{x}_{0}$ and the definition of $\omega_{\alpha}$. Naturally, the recovered values $\widetilde{u}_{h}^{\alpha}$ for $D^{\alpha} u$ at $\mathbf{x}_{0} \in \bar{\Omega}$, are define by

$$
\widetilde{u}_{h}^{\alpha}\left(\mathbf{x}_{0}\right)=I_{1}+I_{2}+I_{3}+I_{4}^{h}+I_{5}+I_{6}^{h},
$$

where

$$
I_{4}^{h}=\int_{B_{+}} u_{h}(x, y) \Delta \psi_{\alpha}\left(\mathbf{x}, \mathbf{x}_{0}\right) d \mathbf{x}, \quad I_{6}^{h}=\int_{\widetilde{B}_{-}} u_{h}(x, y) \Delta \psi_{\alpha}\left(-x, y ; \mathbf{x}_{0}\right) d \mathbf{x} .
$$

The details on the computations of $I_{1}, I_{2}, I_{3}, I_{4}^{h}, I_{5}$ and $I_{6}^{h}$ are given and analysed in [2].

The error term in this case is

$$
\left|D^{\alpha} u\left(\mathbf{x}_{0}\right)-\widetilde{u}_{h}^{\alpha}\left(\mathbf{x}_{0}\right)\right|=\left|\int_{\Omega}\left(u-u_{h}\right) f_{\alpha} d \mathbf{x}\right|=\left|\int_{B_{+}}\left(u-u_{h}\right) f_{\alpha} d \mathbf{x}\right|
$$


where

$$
f_{\alpha}(\mathbf{x})= \begin{cases}\Delta \psi_{\alpha}\left(\mathbf{x}, \mathbf{x}_{0}\right)+\Delta \psi_{\alpha}\left(-x, y ; \mathbf{x}_{0}\right) & : \quad \mathbf{x} \in \widetilde{B}_{-} \\ \Delta \psi_{\alpha}\left(\mathbf{x}, \mathbf{x}_{0}\right) & : \quad \mathbf{x} \in B_{+} \backslash \widetilde{B}_{-} \\ 0 & : \text { elsewhere }\end{cases}
$$

Since $f_{\alpha} \in L^{2}(\Omega)$, if we choose $u_{h}$ to be a piecewise bilinear finite element solution, then the order of convergence will be $O\left(h^{2}\right)$. The bounding procedure is as below.

Find $z \in H_{0}^{1}(\Omega)$ such that

$$
B(z, v)=\left\langle f_{\alpha}, v\right\rangle \quad \text { for } v \in H_{0}^{1}(\Omega)
$$

The finite element solution $z_{h} \in V_{h}^{0} \subset H_{0}^{1}(\Omega) \cap V_{h}$ is found by

$$
B\left(z_{h}, v_{h}\right)=\left\langle f_{\alpha}, v_{h}\right\rangle \text { for } v_{h} \in V_{h}^{0} .
$$

Since $f_{\alpha} \in L^{2}(\Omega)$, by the Lax-Milgram Theorem, there always exist solutions for Equations (40) and (41). The a posteriori pointwise error bound at $\mathbf{x}_{0}$ is determined by

$$
\begin{aligned}
\left|D^{\alpha} u\left(\mathbf{x}_{0}\right)-\widetilde{u}_{h}^{\alpha}\left(\mathbf{x}_{0}\right)\right| & =\left|\int_{\Omega}\left(u-u_{h}\right) f_{\alpha} d \mathbf{x}\right| \\
& =\left|\left\langle f_{\alpha}, u-u_{h}\right\rangle\right| \\
& =\left|B\left(z, u-u_{h}\right)\right|=\left|B\left(z-z_{h}, u-u_{h}\right)\right| \\
& \leq\left\|z-z_{h}\right\|_{E}\left\|u-u_{h}\right\|_{E} \leq \gamma \eta
\end{aligned}
$$

where $\gamma$ and $\eta$ are a posteriori error bounds for $\left\|z-z_{h}\right\|_{E}$ and $\left\|u-u_{h}\right\|_{E}$ obtained by the equilibration procedure proposed in the last section. 


\section{Numerical Experiment}

With the notation defined in the previous sections, we now present some numerical results to verify our theoretical results.

The test problem is

$$
\begin{aligned}
-\Delta u & =f \quad \text { in } \Omega=(0,1) \times(0,1) \\
u & =u_{0} \text { on } \Gamma,
\end{aligned}
$$

with the exact solution

$$
u=u_{0}=10 \sin (2 x+y+2) .
$$

This problem was used in [4]. The finite element approximation is obtained by using piecewise bilinear functions on a uniform mesh of squares of size $h$. The numerical results based on Scheme (10) proposed in [4] calculated at an interior point $\mathbf{x}_{0}=(0.5,0.5)$ are given in Table 1 to compare with our results which are also calculated at $\mathbf{x}_{0}=(0.5,0.5)$ and given in Table 2 . The reliability of the error estimate can be measured by the effectivity index (Index $=$ Error Estimate/True Error) which we would like to be close to 1. It is shown in Table 1 and Table 2 that the effectivity index of Scheme (42) is much more stable and close to 1 than the effectivity index of Scheme (10).

For a point $\mathbf{x}_{0}$ close to or on the boundary, we use Formula (36) to obtain the recovered values. The error bounds can be obtained by using the 


\begin{tabular}{|c|cccc|}
\hline$N$ & $\frac{\partial u_{h}}{\partial x}\left(\mathbf{x}_{0}\right)$ & $\left|\frac{\partial u}{\partial x}-\frac{\partial u_{h}}{\partial x}\right|\left(\mathbf{x}_{0}\right)$ & Error Estimate & Index \\
\hline 9 & -18.316 & $4.13 \mathrm{E}-1$ & $1.54 \mathrm{E}+0$ & 3.72 \\
81 & -18.683 & $4.61 \mathrm{E}-2$ & $4.78 \mathrm{E}-1$ & 10.37 \\
729 & -18.724 & $5.13 \mathrm{E}-3$ & $1.56 \mathrm{E}-1$ & 30.41 \\
\hline
\end{tabular}

TABLE 1: $N=$ number of degrees of freedom, $\epsilon=10^{-4}$, interpolation constant $C_{i}=0.1, \mathbf{x}_{0}=(0.5,0.5)$ and $\frac{\partial u}{\partial x}\left(\mathbf{x}_{0}\right)=-18.729$. Error estimate obtained by using Scheme (10).

\begin{tabular}{|c|cccc|}
\hline$N$ & $\widetilde{u}_{h}^{(1,0)}\left(\mathbf{x}_{0}\right)$ & $\left|\frac{\partial u}{\partial x}-\widetilde{u}_{h}^{(1,0)}\right|\left(\mathbf{x}_{0}\right)$ & Error Bound & Index \\
\hline 9 & -18.134 & $3.88 \mathrm{E}-1$ & $9.65 \mathrm{E}-1$ & 2.49 \\
81 & -18.694 & $3.56 \mathrm{E}-2$ & $6.33 \mathrm{E}-2$ & 1.78 \\
729 & -18.725 & $4.23 \mathrm{E}-3$ & $8.58 \mathrm{E}-3$ & 2.03 \\
\hline
\end{tabular}

TABLE 2: $N=$ number of degrees of freedom, ball radius $R=0.3, \mathbf{x}_{0}=$ $(0.5,0.5)$ and $\frac{\partial u}{\partial x}\left(\mathbf{x}_{0}\right)=-18.729$. Error bound obtained by using Scheme (42).

Schemes (42). The numerical experiments for a point close to the boundary $\mathbf{x}_{0}=(0.05,0.5)$ are shown in Table 3 , and for a point on the boundary $\mathbf{x}_{0}=(0.0,0.5)$ are shown in Table 4 . The observed convergence rates shown in Tables 2-4 are $O\left(h^{2}\right)$ as predicted by our theoretical results. It is also observed that the upper error bounds are realistically tight. 


\begin{tabular}{|c|cccc|}
\hline$N$ & $\widetilde{u}_{h}^{(1,0)}\left(\mathbf{x}_{0}\right)$ & $\left|\frac{\partial u}{\partial x}-\widetilde{u}_{h}^{(1,0)}\right|\left(\mathbf{x}_{0}\right)$ & Error Bound & Index \\
\hline 9 & -17.034 & $1.04 \mathrm{E}-1$ & $6.05 \mathrm{E}-1$ & 5.82 \\
81 & -17.112 & $2.61 \mathrm{E}-2$ & $7.91 \mathrm{E}-2$ & 3.03 \\
729 & -17.135 & $3.16 \mathrm{E}-3$ & $8.58 \mathrm{E}-3$ & 2.72 \\
\hline
\end{tabular}

TABLE 3: $N=$ number of degrees of freedom, ball radius $R=0.3$, $\mathbf{x}_{0}=(0.05,0.5)$ and $\frac{\partial u}{\partial x}\left(\mathbf{x}_{0}\right)=-17.1378$. Error bound obtained by using Scheme (42).

\begin{tabular}{|c|cccc|}
\hline$N$ & $\widetilde{u}_{h}^{(1,0)}\left(\mathbf{x}_{0}\right)$ & $\left|\frac{\partial u}{\partial x}-\widetilde{u}_{h}^{(1,0)}\right|\left(\mathbf{x}_{0}\right)$ & Error Bound & Index \\
\hline 9 & -15.948 & $7.49 \mathrm{E}-2$ & $4.02 \mathrm{E}-1$ & 5.37 \\
81 & -16.015 & $7.64 \mathrm{E}-3$ & $3.65 \mathrm{E}-2$ & 4.78 \\
729 & -16.022 & $9.76 \mathrm{E}-4$ & $3.29 \mathrm{E}-3$ & 3.37 \\
\hline
\end{tabular}

TABLE 4: $N=$ number of degrees of freedom, ball radius $R=0.3$, $\mathbf{x}_{0}=(0.0,0.5)$ and $\frac{\partial u}{\partial x}\left(\mathbf{x}_{0}\right)=-16.0229$. Error bound obtained by using Scheme (42). 


\section{References}

[1] M. Ainsworth and J. T. Oden. A unified approach to a posteriori error estimation using element residual methods. Numer. Math., 65:23-50, 1993. C320, C322, C327

[2] M. Ainsworth, T. Cao and D. W. Kelly. Computable bounds for the error to energy and in quantities of interest in the finite element approximation of non-homogeneous Dirichlet problems. Preprint, 1999. C320, C328, C333

[3] T. Cao, D. W. Kelly and I. H. Sloan. Local error bounds for post-processed finite element calculations. Int. J. Num. Meth. Engrg., 45:1085-1098, 1999. C319

[4] R. Becker and R. Rannacher. A feed-back approach to error control in finite element methods: Basic analysis and examples. East-West J. Numer. Math., 4(4):237-264, 1996. C319, C322, C322, C323, C323, C324, C324, C335, C335

[5] Ju. P. Krasovskii. Properties of Green's function and generalized solutions of elliptic boundary value problems. Soviet Mathematics (translation of Doklady Academy of Sciences of the USSR), 102:54-120, 1969. C323

[6] A. Louis. Convergence for finite element solutions of the Poisson equation. Numer. Math., 33(1):43-53, 1979. C320, C328, C330, C331 
[7] E. Ramm and F. Cirak. Adaptivity for nonlinear thin-walled structures. In D. R. J. Owen and E. Oñate, editors, Computational Plasticity, Fundamentals and Applications, pages 145-163, Barcelona, 1997. CIMNE. C319, C322, C323

[8] S. Wang, I. H. Sloan and D. W. Kelly. Computable error bounds for point-wise derivatives of a Neumann problem. IMA J. Num. Anal., 18:251-271, 1998. C319 